\title{
Subpectoral breast augmentation under local anaesthesia
}

André Camirand MD, Jocelyne Doucet RN

Montreal, Quebec

A Camirand, J Doucet. Subpectoral breast augmentation under local anaesthesia. Can J Plast Surg 1995;3(2):79-82. The procedure for performing subpectoral breast augmentation under local anaesthesia is described, and the results of over 300 operations presented.

Key Words: Anaesthesia, Breast augmentation, Capsular contracture, Subpectoral

\section{Addition mammaire sous-pectorale sous anesthésie locale}

RÉSUMÉ : L'intervention pour addition mammaire sous-pectorale sous anesthésie locale est décrite ici et les résultats de plus de 300 interventions sont présentés.

Over the years, we have performed a large number of subpectoral breast augmentations under local anaesthesia with narcolepsy. We have had great success with a very low percentage of capsular contracture. If given the choice to have the operation done again under local or general anaesthesia, our patients would be unanimous in saying that they would repeat the experience under local anaesthesia.

We prefer the subpectoral approach because most of the blood vessels and nerves are situated between the mammary gland and the pectoral muscles. Thus, we reduce the possibility of damaging these structures. We give antibiotics to our patients the day before surgery, the day of surgery and the day after. We use cephalexin $500 \mathrm{mg}$ every 6 $\mathrm{h}$. If the patient has an allergy to penicillin, we prescribe lincomycin $500 \mathrm{mg}$ every $6 \mathrm{~h}$ for these three days. We believe that protheses implanted behind the pectoral muscle look more natural because they are less spherical and give a pear-shaped appearance with an axillary projection. If there is any capsular contracture, it is better hidden under the muscle.

Table 1 lists the advantages of subpectoral implantation and Table 2 identifies the various factors that help prevent capsular contraction. In very thin people, implanting behind the muscle will avoid palpation of the prosthesis. Sounds resonating from liquid in the mega-cavity or air in the prosthesis will not be heard because of insulation by the muscle. The greatest advantage of placing the prosthesis behind the muscle is probably the 'milking' effect. We know that the smallest surface area for a given volume is a sphere, and this is why capsular contracture develops in a centripetal direction. Every time the muscle contracts it compresses the prosthesis, increasing its surface area and stretching the antagonizing capsular contracture (Figure 1). Finally, we prefer the subpectoral approach because it is easier for us to perform. 
TABLE 1: Advantages of subpectoral implantation

1 Continuous compressing and repeated milking action of the muscle

2 Less bleeding because the vessels pass between the muscle and the breast

3 More vascular medium increases defence against presence of bacteria

4 Axillary projection - pear shaped natural looking breast

5 Cannot palpate the implant in thin patients, especially if there is wrinkling of the prosthesis

6 Isolate the transmission of sound from air in the prosthesis or of liquid in the megacavity at first

7 If there is capsular compression, palpate the muscle instead of the capsular contracture

8 Dissection easier

9 In the presence of ptosis, the muscle compresses the prosthesis (reducing the suprareolar projection) and produces a better axillary projection

TABLE 2: Factors to prevent capsular contraction

A. Compression of the implant (Figure 1)

1 Lie on stomach (For 2 to 3 months)

2 Massage (For 2 to 3 months)

3 Compressive bra (For 2 to 3 months)

B. Less bleeding

1 Subpectoral (blood vessels between the gland and the muscle)

2 Meticulous hemostasis

3 Local anaesthesia, less vomiting and cough

C. Absence of bacteria

1 Asepsis and shampoo, spray opsite on axilla and areola

2 Antibiotics

3 Inframammary approach

D. Megacavity

E. Clean talcum powder from gloves

F. Eliminate silicone bleed

(quality implant for saline inflated implant)

G. Polyurethane or textured implant 


\section{Camirand and Doucet}

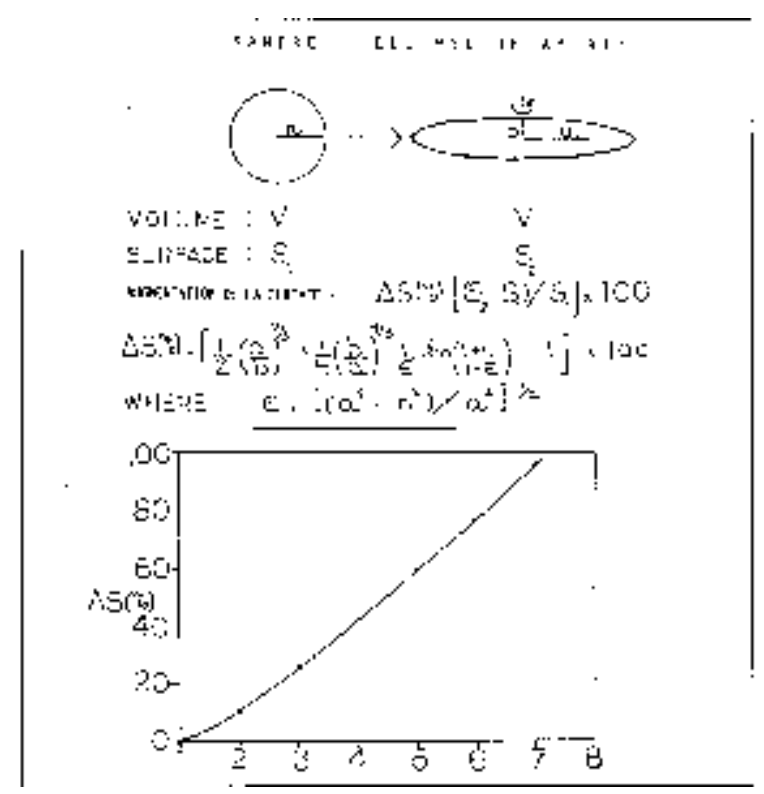

Subpectoral breast augmentation

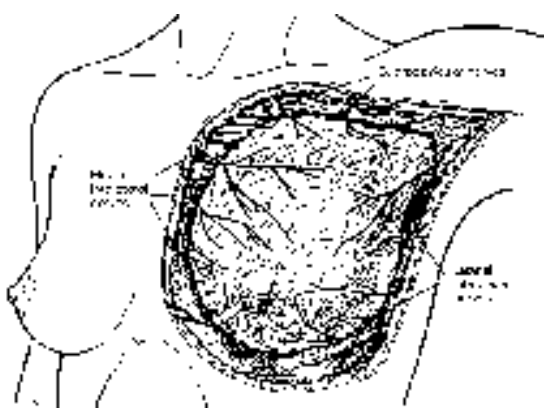

Figure 2) Sensory innervation of the breast and pectoral area. With permission of John Bostwick

Figure 1) The scientific explanation of the value of compressing the prosthesis thereby increasing its surface area and stretching the surrounding capsule to prevent its contracture. Reproduced with permission from Camirand A. Prevention des cocques après augmentations mammaires. Journal de la Société

With anaesthetists being fewer and more difficult to find, local anaesthesia can be a very valuable alternative. A large number of patients resist or want to avoid general anaesthesia. There is less vomiting and coughing after surgery under local anaesthesia, and this reduces the risk of excessive bleeding, another cause of capsular contracture. Finally, patients recuperate faster.

\section{TECHNIQUE}

When patients first arrive they are given $15 \mathrm{mg}$ of diazepam orally. Then, on the operating table, after having prepared our drawings, we inject $2 \mathrm{~mL}$ of fentanyl intravenously over a period of 3 mins with a pulse oximeter. We have used narcolepsy for many years, and we have not had a single incident or problem with this approach.

We spray Opsite on the areolae and in the axilla to prevent contamination from sweating and ducts. We then infiltrate an anaesthetic solution around the gland, horizontally at the level of the inframammary crease along the parasternal and anterior axillary lines and then above, at the level of the second rib into the pectoral muscle (Figures 2,3). The solution used is $50 \mathrm{~mL}$ of xylocaine $1 \%$ with adrenaline and $25 \mathrm{~mL}$ of bupivacaine $0.25 \%$ mixed in $250 \mathrm{~mL}$ of saline. Anaesthetic infiltration is carried out before scrubbing, allowing 20 mins for vasoconstriction. After washing our gloves with water to remove talcum powder, we make a 3 to $4 \mathrm{~cm}$ incision at the level of the inframammary crease. Then, we dissect underneath the pectoral muscle with a finger or scissors. If this manoeuvre turns out too be a little unpleasant for the patient, we do not hesitate to give an extra $1 \mathrm{~mL}$ of fentanyl. 

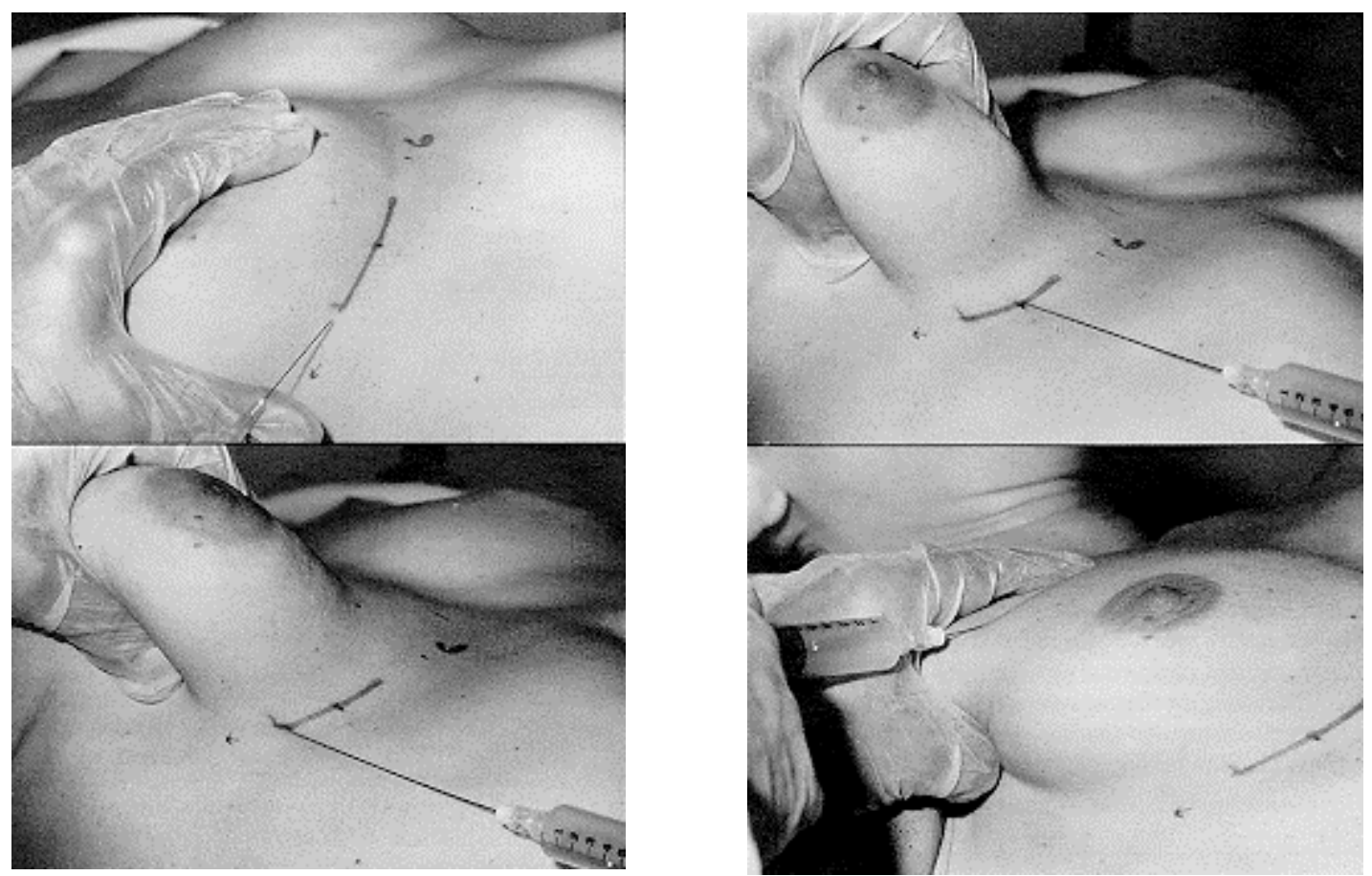

Figure 3) Ring like or circular anaesthesia of the breast and pectoral muscle

Although the majority of patients do not require cauterization, we use a bipolar electrocautery if necessary. After dissecting a megacavity, we explore to make sure there is no bleeding. We then inject $20 \mathrm{~mL}$ of the xylocaine, adrenaline and bupivacaine solution as well as $10 \mathrm{~mL}$ of povidone- iodine in each cavity. We insert the prosthesis, inflate it and suture the deep dermal layer, including the subcutaneous fat to obtain a good eversion of the closure, and then the intradermal layer with Vicryl 4XO. We apply Steri-Strips, compresses and Elastoplasts.

The dressing is removed the next day. Our patients are encouraged to return to their normal activities within a day or two, without any restriction except for movements that could provoke an excessive amount of pain. We do not allow them to practise nordic skiing or windsurfing for eight weeks, because we have had two patients who displaced their prosthesis in a caudal direction after violent contraction of their pectoral muscles. We feel that by returning to their normal activities sooner, not only is convalescence reduced but also the duration of pain is decreased, eliminating muscle spasms. We are convinced that there is not much they can do to cause any damage. We also encourage them to wear a compressive brassiere, and within a day or two after surgery we recommend that they lie on their stomach to compress the prostheses for two to three months, thereby stretching the circumferentially contracting scar to avoid eventual capsular contracture. 

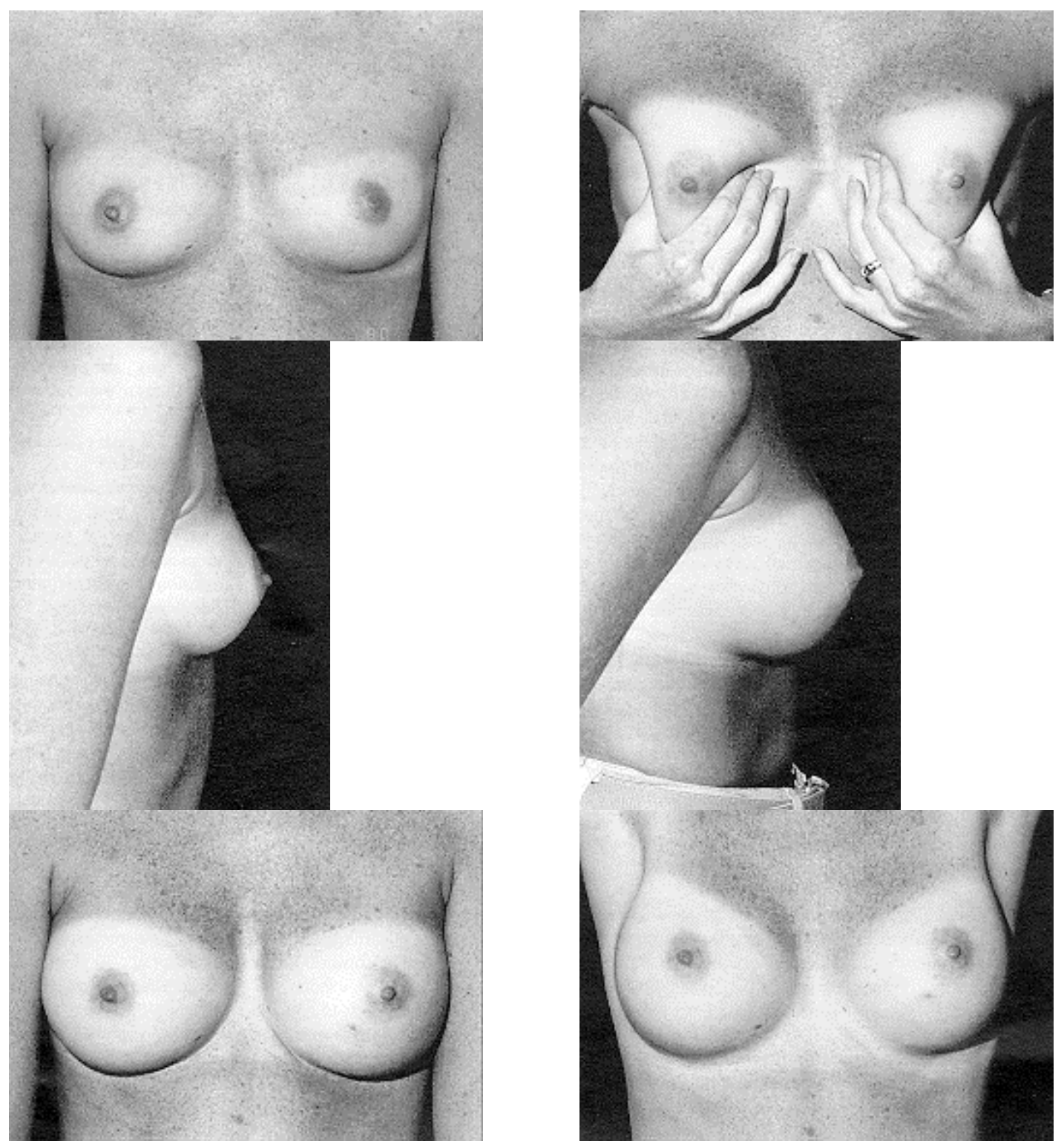

Figure 4) Preoperative (top left, middle left) and one year post subpectoral implantation (bottom left, top right, middle right, bottom right). The breast is soft and looks natural even when the patient raises her arms. Furthermore, the inframammary scars are invisible.

TABLE 3: Complications of 305 primary breast augmentations (average follow-up over 14 months postoperation)

8 hypertrophic scars

5 capsular contractions requiring surgery

4 infections

3 hematomas

2 areolar necrosis following concomitant tuberous breast treatment

1 phlebitis 
CONCLUSION

We prefer the inframammary incision because if it is sutured without tension, the scar is barely visible after a year (Figure 4). If it is, we perform needle dermabrasion (the equivalent of a tatoo without any pigment). We feel that there is less potential contamination with this approach and it is easier to do the inferomedial dissection, thereby reducing the chance of asymmetry. The periareolar and transareolar approaches can produce hyper- or hypopigmented scars that are much more visible, and the risk of contamination is greater. In the axillary approach we find that because the distance between the axilla and inferomedial dissection is considerable, it is much more difficult to control hemostasis and obtain perfect symmetry. Furthermore, axillary sweating during surgery contaminates the wound. We have done over 300 breast augmentations, and so far have had five capsular contractures (Table 3). 